\title{
Neurorehabilitation in upper limb amputation: understanding how neurophysiological changes can affect functional rehabilitation
}

\author{
Lewis A. Wheaton (1)
}

\begin{abstract}
Background: Significant advances have been made in developing new prosthetic technologies with the goal of restoring function to persons that suffer partial or complete loss of the upper limb. Despite these technological advances, many challenges remain in understanding barriers in patient adoption of technology, and what critical factors should be of focus in prosthetics development from a motor control perspective. This points to a potential opportunity to improve our understanding of amputation using neurophysiology and plasticity, and integrate this knowledge into the development of prosthetics technology in novel ways. Here, argument will be made to include a stronger focus on the neural and behavioral changes that result from amputation, and a better appreciation of the time-scale of changes which may significantly affect device adaptation, functional device utility, and motor learning implemented in rehabilitation environments.

Conclusion: By strengthening our understanding of the neuroscience of amputation, we may improve the ability to couple neurorehabilitation with neuroengineering to support clinician needs in yielding improved outcomes in patients.
\end{abstract}

Keywords: Amputation, Neuroplasticity, Prosthetics, Motor learning, Limb loss, Upper limb

\section{Background}

A common concern in neurorehabilitation is understanding how to use neuroscience to shed light on treatment of neurological disease. In many health concerns such as stroke, movement disorders, and cognitive dysfunction the challenge of "how to treat" has been, and continues to be, shaped by focused and relevant neuroscience research. While this is true for large and growing public health concerns, it is also true for other injuries to the nervous system where, by comparison, the rate of injury may be rarer. The rarity of a problem does not relate to the severity of a problem.

As of 2005, of the 1.5 million persons with limb loss (amputees) an estimated 541,000 persons in the United States suffer from some form of upper limb loss, many

Correspondence: law@gatech.edu

School of Biological Sciences, Georgia Institute of Technology, 555 14th Street, Atlanta, GA 30332-0356, USA from trauma, but also public health concerns such as dysvascular disease and cancer [1]. It is projected that by 2020, there will be 2.2 million amputees in the United States. Worldwide estimates are difficult to gather largely due to the variability in why amputation happens and underreporting in the developing world. Amputation does not directly affect the vast majority of people in the United States (or the world), but the impact of amputation is severe and the resources available to amputees are not ideal. For amputees, the use of artificial limbs (prostheses) can become a vital part of their lives. Unfortunately, rejection and non-functional use is high [2]. While futuristic devices are being designed and tested, they are not commonly used by patients, due to the high cost of devices and fiscal policies of coverage and reimbursement for prosthetic technology [3].

Work in Norway focusing on wear patterns amongst a substantial cohort of amputees identifies some concerns [4]. It was found that while amputees that wear 
prostheses tend to have high wear times $(8+$ hours per day), they only use their prostheses for an average of $\sim 50 \%$ of daily living tasks (which included naturally higher numbers for bimanual tasks). In this case, wear does not necessarily relate to use. This study also showed that perceived usefulness scores for a variety of daily living tasks using the most popular prosthetic control mechanisms (body-powered and myoelectric) are markedly similar. Other studies have suggested that lower-cost bodypowered devices are considered sufficient for daily living and just as functional as advanced myoelectric devices [5-7]. These findings are significant policy drivers; many insurers will not authorize reimbursement of myoelectric or other advanced devices unless unique medical necessity exists $[8,9]$. Other approaches such as targeted reinnervation or limb transplantation are available [10] but are invasive, necessitate varying degrees of additional medical burden after amputation, and require more investigation of long-term outcomes [11]. Meanwhile, there is a void of neuroscience research that can inform optimal device design, adaptation and rehabilitation based in neuroscience and motor physiology.

Concepts related to rehabilitation methods and technology in upper limb amputation are not well grounded in empirical data, which has caused concerns about clinical implementation [12]. Despite exciting technological advances in prosthetics, we do not really understand the neurophysiology of amputation and how the motor system may learn and adapt to a new residual and prosthetic limb [13]. The processes of motor learning (both learning how to use the residual limb in its modified state and functional use of the prosthesis) and neuroplasticity (changes within the nervous system to the amputation and integration of prostheses) are vital. Numerous studies of the technological advances in upper limb prosthetics have shown great promise to provide some enhancement to motor control. However, there is a less-frequently discussed issue; there is no technology that has shown immediate sensory establishment of a prosthetic device paired with immediate gains in motor control without a practice-based learning/ adaptation period. This suggests that regardless of the technological advances to date, motor learning is a fundamental principle of prosthesis integration that must be considered. If it is possible that multiple prosthesis technological approaches utilize similar mechanisms of neural plasticity or adaptation, and result in similar improvement or challenges to functional integration, what is the gain of one technological approach over the other? Can a deeper understanding of physiological mechanisms from neuroscience help sharpen prosthetics technology development?

The goal of this review is to highlight our understanding of, and areas where there is a scarcity of knowledge about, the neurophysiology relevant to upper limb loss with a particular focus on neural adaptation, plasticity and motor learning. By doing so, the aim is to emphasize the unique contributions a stronger neuroscientific understanding of the role that amputation may have on adaptations to the nervous system. Further, how motor learning principles can facilitate stronger understandings of the role that sensory feedback may pay in modifying neural circuits for prosthesis adaptation. This will hopefully emphasize the vital role of technological development rooted in a stronger understanding of the underlying neurophysiology of amputation. This review will first focus on known concepts from sensory loss that show potential relevance to amputation, which will serve as a platform for consideration of central nervous system processes that can be affected by amputation. Many studies that demonstrate neuroplasticity following amputation have strong connections to studies using augmented ascending sensory feedback. This has great value for technological approaches that seek to harness sensation and identifying mechanisms that could affect successful implementation. Finally, discussion will focus on how this plasticity may affect motor learning which can have significant impact both development of advanced prostheses and training-based approaches intended to improve prosthesis adaptation and usage.

\section{Concepts from neuroplasticity under sensory loss}

Overall, detailed human neurophysiological studies in amputation are quite rare. There are several methodologies to study neurophysiology that may relate to amputation. Studies of acute perturbations to sensory loss are one example. Modelling acute functional limb loss can be achieved through a variety of techniques, one approach being ischemic nerve block (INB). The use of a blood pressure cuff tourniquet to induce INB has been well evaluated [14-18]. These studies generally demonstrate that INB causes muscle paralysis and rapid plasticity to the limb undergoing INB. Due to the degradation of somatosensory feedback, motor execution relies more on prediction estimates of ongoing limb behavior to supplement diminished proprioceptive input to the somatosensory cortex [19]. While limb paralysis does result from extended INB, prior to paralysis there is no degradation or elimination of motor representations of the limb as identified by remaining descending drive to the deafferented limb [20, 21]. This suggests that INB likely changes how the central nervous system anticipates and plans for motor control and motor accuracy [22]. Mechanistically, data suggests that the motor cortex contralateral to INB increases in excitability through rapid down regulation of gamma-aminobutyric acid (GABA)-related inhibitory circuits [23].

Important for relevance to amputation and rehabilitation, the effects of limb loss are not limited to the 
contralateral hemisphere. Werhahn and colleagues revealed increased motor excitability to the limb opposite INB, which could be inhibited by the GABA-A agonist lorazepam [24]. Due to the GABA-ergic modulation, it was proposed that rapid ipsilateral cortical plasticity promotes cortical excitability to the contralateral limb (i.e., limb opposite INB [25-27]). More on this from the perspective of amputation will follow, though it may be important for understanding motor learning in the amputated limb, and the unaffected limb. From a motor control perspective, INB of the right hand caused transient increased grip strength, tactile discrimination and sensibility in the left hand [28]. Tactile spatial acuity and somatosensory processing also increase in the limb contralateral to deafferentation [25].

Using whole-brain neuroimaging techniques, we can illustrate neural changes that suggest plasticity in sensory systems can influence motor control beyond motor cortex. Using functional magnetic resonance imaging (fMRI), we sought to understand the neural systems involved in changing the ability to use visual or somatosensory feedback by selectively reducing the reliability of each sense individually during a tool use task [29]. Participants interacted with a task board to use tweezers to "lift and place" an orange block with the right hand. Participants performed the task with intact visual and proprioceptive sensation, then with reduced vision (with partially occluded glasses), and finally reduced sensation of the right hand with INB. Findings showed that during the task with intact vision and sensation (Fig. 1a), expected left sensorimotor areas were predominantly involved, which increased during reduced vision (Fig. 1b,d). Reduced sensation as a result of INB significantly diminished the activity in left sensorimotor areas and cerebellum, (Fig. 1d) caused increased posterior parietal and occipitotemporal complex activation (Fig. 1f). These areas have been shown to be highly involved in tasks that require hand-eye coordination [30-32], which may be useful in overcoming altered sensorimotor states of the limb that may impair formation of the motor plan. This supports the proposal that lost/impaired proprioception can induce a significant change in sensory weighting for motor control beyond motor cortex [22].

The use of sensation is a vital component for theories of upper limb motor control. Studies have suggested a dual pathway for action based on the forward/inverse model concept [33]. Such models anticipate sensory consequences of upcoming actions. In order for an accurate prediction of the action goal, predicted sensory information is compared to actual sensory information [34, 35]. These sensory-based models have been proposed to be useful for predicting motor outcomes [36]. Relating this to amputation, loss of sensory feedback may perturb the ability to use sensory-based models to function in action prediction and motor planning. In a study done by Moisello and colleagues, 12-h of upper limb immobilization was shown to affect behavioral coordination of limb segments and delayed movement onset, suggesting impaired feedforward control [37]. However, loss of proprioception need not completely impair sensory-based models of motor control, as sensory feedback is also acquired from the visual system [38]. Amputees are capable of

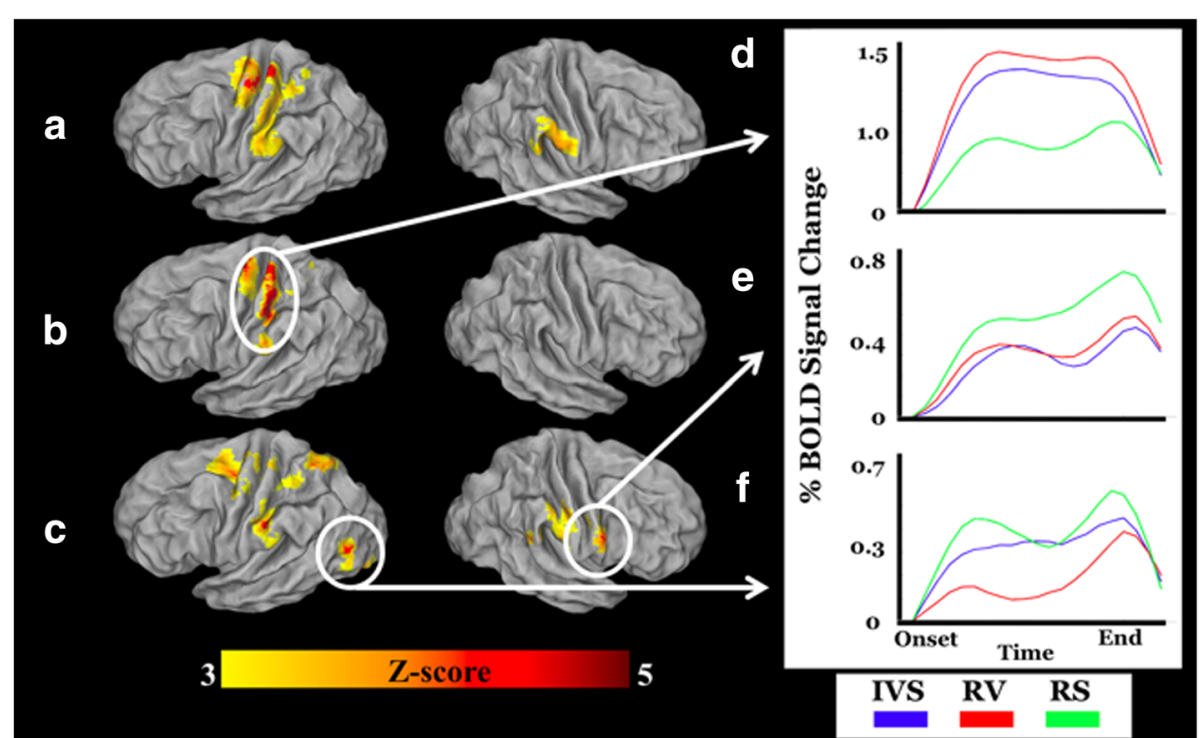

Fig. 1 Cortical fMRI activations observed for (a) intact sensation, (b) reduced visual reliability and (c) reduced somatosensory reliability. Time course representations, in percent signal change relative to the mean BOLD response, for three regions of interest: $\mathbf{d}$ left sensorimotor cortex, e left lateral occipital cortex and (f) right sensorimotor cortex (from [29], with permission from the publisher) 
interacting with stimuli in peripersonal space, such that their visuospatial representations for motor control are intact [39]. Additionally, previous work has demonstrated that amputees are capable of grip selection planning in their amputated limb, suggestive of the use of internal representations of actions that are shared from the intact limb [40]. Thus, it seems possible that even without bionic prostheses, the motor system may adapt using sensory estimates from alternative sources [19]. Unfortunately, we do not know the scale of neural adaptations that accompany amputation. In line with our previously described fMRI evaluation of sensory weighting [29], recent evidence suggests a putative visual adaptation that may have great relevance to behavior and rehabilitation.

\section{Neurobehavioral adaptations following amputation Cortical plasticity}

Unlike central nervous pathology (such as stroke where a lesion disrupts local and regional brain function), traumatic amputation is thought to not commonly damage the structure of the cerebrum (there is debate about cortical and white matter anatomical changes [41-43] which, if present, appear to better correlate with phantom sensations than motor function [44]). Substantial functional changes have been shown to occur in amputation indicative of neuroplasticity. It is clear that amputation affects motor cortex function by promoting an expansion of the residual limb segments into the former limb territory [45-48]. Many studies have looked at the reorganization of the nervous system after amputation, but commonly in the perspective of phantom sensations (e.g., pain), which are likely result of ongoing neuroplasticity [49-52]. Further studies have shown the relevance of phantom sensations in relation to cortical plasticity. Strong relationships have been seen correlating phantom sensations and the degree of cortical reorganization [53, 54]. Further, approaches to manage phantom pain could serve to aid in sensory reorganization $[55,56]$. As will be described in later sections, establishment of sensation from the prosthetic device likely plays a pivotal role in cortical plasticity that can affect motor learning processes.

Studies in animals have revealed mechanisms of complex plasticity that has a significant role in motor system physiology. Residual cortical limb representations may affect ongoing plasticity within cortical regions. Studies in adult rats with forelimb amputations after birth revealed that when forelimb stump representations in the contralateral somatosensory cortex was inactivated (or reduced [57]), principally hindlimb inputs were revealed. This suggests that forelimb stump representations can actively suppress the expansion of hindlimb inputs [58]. This seemingly proposes a similar mechanism thought to be involved in human studies using INB acutely [23]. Using positron emission tomography,
GABA receptor binding was studied in unilateral amputees years following injury. Results showed increased GABA receptor binding in upper limb regions of the primary sensorimotor areas bilaterally, which suggests long-term stability of cortical reorganization of the stump and former limb territory [59].

Recent studies report large-scale changes in neural networks including and beyond sensorimotor cortex following amputation [60]. This work takes advantage of functional connectivity of sensorimotor areas during resting state, avoiding confounds induced by specific motor tasks. The authors identified a progressive disconnection of the missing hand cortex and the sensorimotor network, with a concomitantly stronger connection with the so-called default mode network [61]. While it is unclear what is driving this network plasticity, it does reflect a functional shift of the entire sensorimotor network that could have rehabilitative consequences. In a case-study report, it was shown that amputees may recruit areas outside of the expected left parietofrontal system for motor-relevant tasks (attributed to the mirror neuron system), including the right temporoparietal junction (attributed to the mentalizing system [62]) [63]. This may correspond to clinical observations suggesting that amputees develop a behavioral adaptation of placing a greater emphasis on the visuomotor aspects of motor task performance $[64,65]$. We have suggested that neural patterns seen in electroencephalography (EEG) reflect widespread changes in neural oscillations in amputees [66]. In this work, amputees performed a basic motor task with their amputated (right) arm, and then their sound limb to evaluate neuroplasticity that may occur following amputation. Effects were compared against a population of leftand right-handed persons with sound limbs. Results demonstrated that for movements with the sound limb amputees showed activation over the contralateral motor cortex that was greater than the persons with sound limbs. Movements with the amputated limb showed bilateral (and mainly right) parieto-occipital activation (Fig. 2). These examples help to illustrate the complex neural changes that follow in the cortex, which in the motor system may be influenced by changes in activation of projection areas to sensorimotor cortex [67]. However, argument can be made that cortical plasticity is a result of plasticity outside of the cortex.

\section{Subcortical influences on cortical plasticity}

Very few studies have investigated the role of subcortical reorganization on motor function related to upper limb loss. Studies in animal models have well demonstrated changes in deep brain structures that may impact cortical plasticity. These have focused on the sensory reorganization that can occur following limb loss by studying subcortical nuclei that relay ascending sensory 


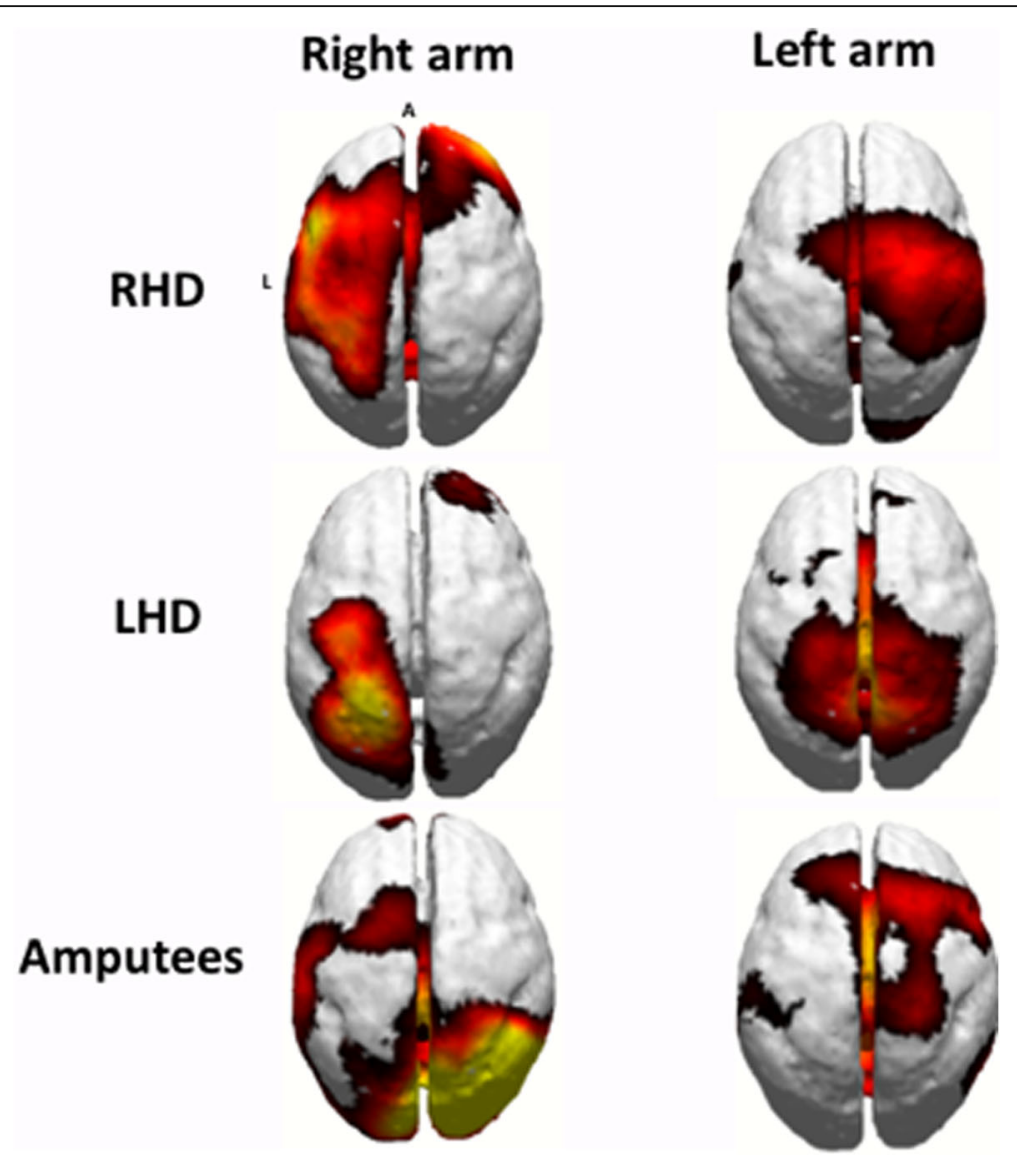

Fig. 2 Current source density of beta band (18-22 Hz) activity during motor planning in each group (from [66], with permission from the publisher)

information to higher-order areas. Of emphasis is to attempt to determine what subcortical areas might serve as a substrate for cortical reorganization. In the case of the upper limb, the posterior medial lemniscus pathway carries sensory information from the upper part of the body. Once peripheral axons enter the spinal cord, most branch to ascend into the dorsal columns and traverse into the medulla where a synapse is made on the cuneate nuclei. Relay neurons send projections to the ventral posterior nucleus of the thalamus, which sends information into the primary somatosensory cortex. Thus, possible brain regions for sensory cortical reorganization based on this projection may occur in the spinal cord, cuneate, thalamus, and/or intrinsic to the cortex. Studies of monkeys have demonstrated changes to motoneurons and spinal cord atrophy in the residual limb that could relate to cortical changes following amputation $[68,69]$. Forelimb amputation in rats has demonstrated that over the course of 30 weeks post amputation, very little new sensory input is seen at the cuneate nucleus in the zone of former forepaw territory, and that the main reorganization occurs around the former forepaw zone [70]. This is despite the finding that shoulder input occupies the former forepaw barrel cortex within 4 weeks of amputation. This suggests that cuneate reorganization (including inputs into the cuneate [71]) is not related to cortical reorganization. Further studies revealed that cortical reorganization may be related to changes in ventral posterior lateral nucleus of thalamus (VLP), via latent subthreshold cuneothalamic residual limb inputs in the former limb territory [72]. This largely concurs with a prior study in neonatal forelimb removal in rats, showing that abnormal hindlimb receptive fields in the stump territory of primary sensory cortex is attributed to an increase in VPL neurons that have sensory receptive fields on the stump and hindlimb [73]. The resultant dual hindlimb-stump representation was not attributed to changes in intracortical connections within, or changes to thalamocortical projections to, somatosensory cortex. This suggests that cortical plasticity may have a thalamic origin, which, given the robust density of thalamocortical projections to motor cortex may suggest that if sensation is lost, thalamocortical plasticity may have profound impacts on cortical substrates for learning [73]. Studies have demonstrated that thalamocortical projections (notably from ventral anterior/ventral lateral thalamus 
(VA/VL) complex) into motor cortex have populations of neurons that show motor learning plasticity, by demonstrating biased activation towards learned tasks [74].

These studies identify the significant role that the thalamus (and thalamocortical pathways) play in shaping cortical plasticity after amputation, and suggest a role in motor learning. While it is presently unclear what role limb loss may have on VA/VL complex specifically, given widespread projections of these nuclei to frontal premotor areas [75], thalamocortical networks arising from VA/VL nuclei may have significant importance on how cortical networks involved in motor learning function following limb loss. While the VL thalamus sends efferents to primary motor cortex (ventral anterior nucleus of thalamus, principle segment; VLp), it also has dense connectivity to premotor (ventral anterior nucleus of thalamus, anterior segment; VLa) and posterior parietal (VL) cortices [76, 77]. Studying markers of glia cell activation, it was seen that increases in thalamic (but not sensorimotor cortical) glial signaling persisting years after nerve injury [78]. This finding further supports the hypothesis that thalamic pathways are vital to consider, and the role they play in functional plasticity long after injury. Further, these changes may differ based on the stage of development that amputation occurs [79]. While evidence is lacking specifically as to the nature of the role of VL in human neurophysiology and amputation, some relevance may be seen in studies detailing changes in beta-band oscillations at a thalamocortical network level. Physiological changes seen in cortical recordings demonstrating neuroplasticity using EEG [66] may be of thalamic origin due to the coherence between thalamus and cortex within specific frequencies of activation [80].

\section{Relevance of neuroplasticity to motor learning}

What is the importance of cortical and subcortical neuroplasticity to motor learning in amputation? Regardless of the prosthesis device designs, motor learning is a vital component [81]. As previously discussed, significant technological advancements in prosthetics have yet to provide a device that can fully and immediately provide sensory establishment and motor control improvement without a practice-based learning/adaptation period. As described above, there is vast plasticity of thalamocortical circuits following amputation. It is possible that the development of prosthetic limb technology must consider how, or if, this underlying neuroplasticity is a challenge to prosthetic device learning and adaptation. There is evidence emerging in behavior and neurophysiology that underscores the significance of neuroplasticity and adaptation in motor learning for amputation. However, technology that relays sensory feedback from the prosthesis to the body appears not to be a requisite for motor control improvement.

\section{Concepts with engineered prosthetic limb sensory feedback}

Using brain machine interface approaches, it has been demonstrated that stable connectivity between primary cortex and the striatum is pivotal for skill learning with prostheses [82]. While this particular study focused on neuroprosthetics, the concept that there has to be stability between corticostriatal areas to provide a stable neural platform for motor learning is valuable. As we have reviewed, it seems evident that ascending sensory loss is capable of promoting cortical plasticity in motor cortex, the integrity of primary motor-striatal pathways in amputation becomes a pivotal issue in understanding neuroplastic changes that may impact prostheses that rely on neural signals. It is unclear what occurs in the striatum after amputation, but immobilization studies show a use-dependent function of motor activation. Immediately upon removing chronic immobilization in the upper limb, decreases in striatum activity is seen which can be recovered as motor control recovers [83]. Continued studies further isolated this effect to the putamen, showing that loss of putamen activation relates to loss of learned movement sequences during chronic immobilization [84]. These studies highlight the possibility that functional disuse can lead to maladaptive plasticity within the striatum, which could have negative consequences for motor learning. In the case of immobilization, removal of the orthotic gives rise to the natural limb and the pre-immobilization state. This return to the natural limb state is not possible in the case of amputation. Thus, understanding how prosthetic devices with sensory feedback may unmask this potential striatal-cortical maladaptive plasticity to encourage learning is a pivotal step in understanding prosthesis adaptation and motor learning.

Physiologically, research has demonstrated a critical role of proprioception in shaping motor cortex plasticity. Using transcranial magnetic stimulation, input-output curves and intracortical inhibition were measured before and after $10 \mathrm{~h}$ of limb immobilization [85]. Participants were placed in one of 3 groups, tactile vibration, proprioceptive vibration (yielding illusory movements), and a no-vibration control. Results showed that the absence of vibration caused a decrease in contralateral motor cortical excitability, but proprioceptive vibration (and to some degree tactile vibration) minimized this effect. Ipsilateral motor cortex showed hyperexcitability in the absence of vibration and tactile vibration groups, but did not show hyperexcitablilty under the proprioception vibration group. This result suggests that the absence of proprioception (and not just the loss of movement in immobilization) may cause hemispheric imbalance of excitability, which may pave the way for motor functional loss of the cortical motor representation. This suggests that ascending sensory feedback (through a 
cuneo-thalamo-cortical projection) can modify motor plasticity. This has strong relevance to amputation rehabilitation as it is unclear what the return of proprioception (via haptic feedback prosthetics) would do to potentially unmasking hemispheric imbalance seen chronic upper limb amputees [86, 87]. While motor learning may promote cortical excitability [88], studies have revealed that decreased excitability of primary cortex can suppress skill acquisition with the contralateral hand, while promoting learning with the ipsilateral hand [89]. Such a pattern could influence motor learning with prostheses without proprioceptive or haptic feedback. Behavioral evidence shows that in body-powered prosthesis users, motor learning and recall is significantly affected by the presence of proprioceptive motor errors (e.g., slips, drops) in a tactile visuomotor learning task [90]. In contrast, persons performing the task with sound limbs show reductions of error, and increased learning. This may suggest that proprioceptive feedback beyond haptic feedback is helpful to the learning process.

Sensation can be established with training using prostheses. Sensory representations of functional robotic hands can develop after $30 \mathrm{~h}$ of tactile feedback-based training on using motoric tasks with a robotic limb [91]. Evoked tactile sensation methods have demonstrated the possibility of evoking sensory perceptual thresholds as a finger map on the radial and ulnar sides of the residuum of forearm amputees, which could serve as a way to encode sensory feedback through training [92]. Targeted reinnervation approaches for prosthetics have demonstrated cortical remapping that suggests a return of sensorimotor control closer to their non-amputated state [93]. In a retrospective study, targeted reinnervation shows success in establishing sensory perception in the prosthetic limb, yet significant variability across persons exists [94]. These sensory based outcomes could be highly related to motor function improvements in persons with limb loss that are able to have sensory and motor reinnervation $[95,96]$. As a recent example, advances have shown promise to implantable (and potentially wireless, [97]) electromyography systems that are capable of driving a prosthetic hand with upwards of $3^{\circ}$ of freedom or isolated finger movements [98-100]. However, recent work evaluating motor unit activity revealed motor units that covered smaller surface areas (with high degree of overlap) and shorter action potential durations in amputees with targeted reinnervation compared to persons with sound limbs [101]. This could explain the difficulty in simultaneously coordinating $>3^{\circ}$ of freedom after targeted reinnervation [102], and stress the importance of a stronger understanding of motor unit physiology after reinnervation.

Exciting new work is highlighting the capacity to provide information about the intensity of sensory feedback capability to amputees with peripheral nerve electrodes [103]. However, as we will discuss below, rehabilitation paradigms to deal with the neuroplasticity from lost proprioception may also support motor learning with prostheses that do not have sensory feedback available. Indeed, it has been demonstrated that the introduction of vibrotactile feedback while training with prostheses has led to remarkable variability in motor outcomes including no benefit/maladaptive [104], modest benefit $[105,106]$, to significant benefit that was seen after prolonged training [107]. Such evidence should cause researchers to consider what the unique role of artificial sensory feedback might have on the nervous system, and whether improvements are driven by a training effect that may, or may not be, comparable with more rudimentary devices.

\section{Concepts without engineered prosthetic limb sensory feedback}

Restoring sensation through advanced prostheses that intentionally provide sensory feedback may not be the only option. Indeed, sensation can be acquired through body powered devices, as position and vibration of the cabling support provides position and force information [108]. This mechanical information can be harnessed to support object identification [109]. This may relate to how non-biological implements (such as tools) can obtain a sensorimotor embodiment after training [110]. In this case, neurons of the ventral premotor cortex of the monkey that fired to fingers additionally fired to seen actions of learned pliers. It is possible that sensory feedback obtained during training of the pliers afforded this process. In the case of amputation, motor "entrainment" without direct sensation has been seen in the so called "rubber hand illusion" $[111,112]$ where stimulation of a rubber hand activates parietofrontal areas in observers that have developed stimulus synchrony with the rubber hand [113-115]. Participants will even report a sense of ownership with the rubber hand [116], and can develop identity with a supernumerary hand without a loss of ownership of their own hand [117]. Evidence of identity with artificial hands, even those that reshape the human body (e.g., supernumerary hands), is critical to consider as it suggests that ownership is not essentially tied to the actual presence of ascending sensory feedback (i.e., feedback delivered through an engineered haptic feedback system in the rubber hand/prosthesis) but merely the ability to translate non-biological componentry into body schema that may involve some level of learned sensory assimilation.

This opens the possibility that training paradigms may sufficiently enable sensorimotor embodiment of prostheses for motor control with [91] or without sensory feedback from a prosthetic device. In an early neural study [118], we sought to identify if sensorimotor areas 
might be sensitive to various approaches to motor learning. Chiefly in the case of amputation, learning motor skills with prostheses may be difficult to learn from persons with sound limbs, compared to learning with another prosthesis user based on differences in limb state between the observer (amputee) and the observed (trainer). Persons with sound limbs and traumatic upper limb amputee prosthesis users imitated actions of other prosthesis users or intact limbs. All of the amputees were "low users" of prostheses, indicated by average time device was worn per day $(\sim 4.4 \mathrm{~h} /$ day $)$ and self-report. Results showed that when amputees imitate intact subjects, they have a persistent bilateral occipito-parietal and right temporoparietal positivity in motor planning. However, when amputees emulated other amputees, they showed an emergent left parietal and mesial frontal activation during motor planning. The consequence of right hemispheric activation in the amputation group might reflect heightened visuospatial needs in learning to translate action of the sound limb into appropriate motor outcomes (as demonstrated in tool use in healthy adults [119-122]). It is known that amputees face increased difficulty in motor imagery [123], which may affect interfere with developing motor skills in rehabilitation. This is would be critical barrier to skill acquisition and would demonstrate that dissimilarity of limb state is a challenge to encoding action skills in a sensorimotor reference frame in observational rehabilitation. It is also possible that non-invasive brain stimulation may also help support entrainment of motor patterns to help support motor learning [124], with potential applications to using prostheses [125]. This could be of strong benefit to support training to establish proper neural circuits to improve motor skill coordination with prostheses.

Based on our prior work [118], we have proposed a possible neural adaptation modeled in persons with sound limbs relevant to observation-based rehabilitation training. We utilized a fictive model of amputation [126] to longitudinally assess neurobehavioral adaptations to learning prosthesis use based on learning with a prosthesis user ("matched limb") versus a person with sound limbs ("mismatched limb"). Immediate to fitting with the prosthesis, and on the first day of training, persons in the matched limb group showed dominant left parietofrontal activation patterns. However, persons in the mismatched group showed heightened occipital and parietal activity bilaterally, with decreased mesial premotor/ motor activity. These patterns held stable through training. This suggests that proper training may promote sensorimotor activation patterns for prosthesis use, which may better enable functional adaptation to occur. We have identified similar findings in kinematics, demonstrating improved motor control outcomes with matched limb training $[126,127]$.

\section{Other relevant approaches}

Research is also emphasizing training concepts that could be relevant to amputees prior to prosthesis fitting. Studies have proposed the idea of bilateral transfer of motor skills across limbs using a prosthesis simulator [128]. The motivation of this idea is to take advantage of learning that can be done in the unilateral amputee's sound limb that may promote motor skill learning in the weeks to months before they can be fit with a prosthetic limb. For one example, therapies that focus on interlimb transfer and training of skilled use of the residual limb for future prosthesis usage $[129,130]$ may be advantageous to start training directly after amputation and promote prosthesis-based motor plans in the affected cortex. Research has shown that the "deprived cortex" (contralateral to the amputation) is used by whichever limb individuals are using most, suggesting mechanisms of experience-driven plasticity shaping bilateral sensorimotor reorganization [131]. Such experience-dependent plasticity may be harnessed in motor learning. This is valuable, as training that promotes use of the residual limb should be of emphasis. It may be that such training focus could do well in limiting putative maladaptive cortical and subcortical plasticity. In one example, the use of virtual reality training may enhance affected limb use before fitting to optimize outcomes. While this concept has been approached with advanced prostheses [132], it may also extend to all forms of devices.

Pharmacological interventions in amputees may influence motor control and motor learning. For example, phantom limb pain has shown resolution with duloxetine (serotonin-norepinephrine reuptake inhibitor) and pregabalin (increases GABA production) [133]. It has been noted that duloxetine may have benefits to psychomotor performance [134]. However, it is argued that decreases in local GABA are beneficial to motor learning related increases in activity in primary motor cortex [135] while increases can suppress motor learning [24] which may suggest a negative effect of pregabalin. Pain control after amputation can involve prolonged use of many pharmacological agents including anticonvulsants, tricyclic antidepressants, and even electrical nerve stimulation [136]. An improved understanding of the role these agents may play in motor learning and neuroplasticity are vital in developing empirical evidence in future studies, particular in animal studies where neuropharmacology can be better evaluated at a cellular and systems level.

\section{Conclusions}

The complexity of neural changes following amputation that may have profound impacts on motor planning, execution and learning offer unique opportunities to better inform rehabilitation strategies in the future. 
Understanding therapies and technology through the lens of whether these approaches can unmask or utilize ongoing neuroplasticity has great value. Notably, evidence that neuroplasticity, "embodiment" and motor learning may develop without restorative sensory feedback from prostheses helps illustrate the complex neuroanatomy and physiology that can contribute to challenges related to upper limb loss. Training and practice are a core aspect of all motor improvements seen with advanced bionic and more rudimentary prostheses. Specifically, these observations should drive the neuroscience and neuroengineering community to collaboratively focus more on what factors might enhance plasticity to restore function, and whether various forms of sensory feedback provide common or distinct mechanisms to improved function, neural plasticity and adaptation. This type of evidence is critical to demonstrating the unique capacities for certain technologies in restoring function, and provide a richer literature to support clinical integration. As well, while not specifically covered in this review, a vital concept is understanding how control interfaces (body bowered versus myoelectric), physical factors such as limb length, surgical methods and socket design (particularly important for myoelectric designs) augment neural adaptations and influence motor learning [3, 41, 137-139]. Further, the fundamentals of motor control in amputees (e.g., functional lateralization of the sound limb or the affected limb with prostheses, how sensory feedback is used in affected and unaffected limbs) remains enigmatic. Understanding how these concepts work with device designs and emerging technology (such as sensory-based prostheses and even supernumerary limbs) can enable a stronger integration of neuroscience, neuroengineering, and clinical research (as has been well implemented in other rehabilitation models, such as stroke [140]) to yield stronger scientific and technical knowledge to improve outcomes. In discussing evidence-based approaches to prosthetic rehabilitation [12], the authors well state the challenges of integrating fast-moving technological designs into the clinic based on evidence that can be difficult to interpret, and lacking in rigor for health-care financers. This is a fundamental challenge. While "research" (in [12], this more strongly, but not exclusively means technological development) is needed to offer solutions on device designs, it is possible that collaborative neuroscience and neurorehabilitation approaches may shape both training methodology and technological development. Answers to questions about the necessity of sensory feedback, motor learning with prostheses, neuroplasticity, and needs of the patient mutually inform each other to shape a deeper and relevant knowledge base for rehabilitation. Thus, a neuroscience, neuroengineering, and clinical collaborative/informative model will likely help support a stronger future for shaping positive rehabilitation outcomes. This is essentially done by building collaborations across these communities dedicated to evidence-informed practice that can become the basis for fundamental changes in rehabilitation.

\section{Abbreviations \\ EEG: Electroencephalogaphy; fMRI: Functional magnetic resonance imaging; GABA: Gamma-aminobutyric acid; INB: Ischemic nerve block; VA: Ventral anterior nucleus of thalamus; VAa: Ventral anterior nucleus of thalamus, anterior segment; VAp: Ventral anterior nucleus of thalamus, principle segment; VL: Ventral lateral nucleus of thalamus; VLP: Ventral posterior lateral nucleus of thalamus \\ Acknowledgements \\ I wish to thank Dr. T. Richard Nichols for helpful discussion in forming this review. \\ Funding \\ The author wishes to acknowledge generous support from the National Science Foundation (NSF: 1328567), John Templeton Foundation (47994) and the Cullen-Peck Faculty Fellowship.}

\section{Availability of data and materials \\ Not Applicable.}

Authors' contributions

LAW wrote the work.

\section{Competing interests}

The author declares that he/she has no competing interests.

\section{Consent for publication}

Not Applicable.

Ethics approval and consent to participate Not Applicable.

\section{Publisher's Note}

Springer Nature remains neutral with regard to jurisdictional claims in published maps and institutional affiliations.

Received: 8 September 2016 Accepted: 15 May 2017

Published online: 22 May 2017

\section{References}

1. Ziegler-Graham K, MacKenzie EJ, Ephraim PL, Travison TG, Brookmeyer R. Estimating the prevalence of limb loss in the United States: 2005 to 2050. Arch Phys Med Rehabil. 2008;89(3):422-9.

2. Datta D, Selvarajah K, Davey N. Functional outcome of patients with proximal upper limb deficiency-acquired and congenital. Clin Rehabil. 2004;18(2):172-7.

3. Resnik L, Meucci MR, Lieberman-Klinger S, Fantini C, Kelty DL, Disla R, Sasson N. Advanced upper limb prosthetic devices: implications for upper limb prosthetic rehabilitation. Arch Phys Med Rehabil. 2012;93(4):710-7.

4. Ostlie K, Lesjo IM, Franklin RJ, Garfelt B, Skjeldal OH, Magnus P. Prosthesis use in adult acquired major upper-limb amputees: patterns of wear, prosthetic skills and the actual use of prostheses in activities of daily life. Disabil Rehabil Assist Technol. 2012;7(6):479-93.

5. Biddiss E, Chau T. Upper-limb prosthetics: critical factors in device abandonment. Am J Phys Med Rehabil. 2007:86(12):977-87.

6. Berke GM, Fergason J, Milani JR, Hattingh J, McDowell M, Nguyen V, Reiber GE. Comparison of satisfaction with current prosthetic care in veterans and servicemembers from Vietnam and OIF/OEF conflicts with major traumatic limb loss. J Rehabil Res Dev. 2010;47(4):361-71.

7. Dudkiewicz I, Gabrielov R, Seiv-Ner I, Zelig G, Heim M. Evaluation of prosthetic usage in upper limb amputees. Disabil Rehabil. 2004;26(1):60-3.

8. Manual WHP. Myoelectric Prostheses for the Upper Limb. In; 2015. 
9. Policy CMC: Myoelectric Upper Extremity Prosthetic Devices. In: CG-OR-PR-05; 2014-2015.

10. Carlsen BT, Prigge P, Peterson J. Upper extremity limb loss: functional restoration from prosthesis and targeted reinnervation to transplantation. J Hand Ther. 2014;27(2):106-13. quiz 114.

11. Gart MS, Souza JM, Dumanian GA. Targeted muscle reinnervation in the upper extremity amputee: a technical roadmap. J Hand Surg [Am]. 2015;40(9):1877-88.

12. van Twillert S, Geertzen J, Hemminga T, Postema K, Lettinga A. Reconsidering evidence-based practice in prosthetic rehabilitation: a shared enterprise. Prosthet Orthot Int. 2013;37(3):203-11.

13. Sawers A, Hahn ME, Kelly VE, Czerniecki JM, Kartin D. Beyond componentry: How principles of motor learning can enhance locomotor rehabilitation of individuals with lower limb loss-a review. J Rehabil Res Dev. 2012;49(10):1431-42.

14. Baron GC, Irving GA. Effects of tourniquet ischemia on current perception thresholds in healthy volunteers. Pain Pract. 2002;2(2):129-33.

15. Bjorkman A, Rosen B, Lundborg G. Acute improvement of hand sensibility after selective ipsilateral cutaneous forearm anaesthesia. Eur J Neurosci. 2004;20(10):2733-6.

16. Lundborg G, Bjorkman A, Hansson T, Nylander L, Nyman T, Rosen B. Artificial sensibility of the hand based on cortical audiotactile interaction: a study using functional magnetic resonance imaging. Scand J Plast Reconstr Surg Hand Surg. 2005;39(6):370-2

17. Bjorkman A, Rosen B, Lundborg G. Enhanced function in nerve-injured hands after contralateral deafferentation. Neuroreport. 2005:16(5):517-9.

18. Schlee G, Milani TL, Sterzing T, Oriwol D. Short-time lower leg ischemia reduces plantar foot sensitivity. Neurosci Lett. 2009;462(3):286-8.

19. Christensen MS, Lundbye-Jensen J, Geertsen SS, Petersen TH, Paulson OB, Nielsen JB. Premotor cortex modulates somatosensory cortex during voluntary movements without proprioceptive feedback. Nat Neurosci. 2007;10(4):417-9.

20. McNulty PA, Macefield VG, Taylor JL, Hallett M. Cortically evoked neural volleys to the human hand are increased during ischaemic block of the forearm. J Physiol. 2002;538(Pt 1):279-88.

21. Reilly KT, Schieber MH, McNulty PA. Selectivity of voluntary finger flexion during ischemic nerve block of the hand. Exp Brain Res. 2008;188(3):385-97.

22. Imamizu H, Kawato M. Brain mechanisms for predictive control by switching internal models: implications for higher-order cognitive functions. Psychological Research. 2009;73(4):527-44.

23. Ziemann U, Hallett M, Cohen LG. Mechanisms of deafferentation-induced plasticity in human motor cortex. J Neurosci. 1998;18(17):7000-7.

24. Werhahn KJ, Mortensen J, Kaelin-Lang A, Boroojerdi B, Cohen LG. Cortical excitability changes induced by deafferentation of the contralateral hemisphere. Brain. 2002;125(Pt 6):1402-13.

25. Werhahn KJ, Mortensen J, Van Boven RW, Zeuner KE, Cohen LG. Enhanced tactile spatial acuity and cortical processing during acute hand deafferentation. Nat Neurosci. 2002:5(10):936-8.

26. Neumann-Haefelin T, Hagemann G, Witte OW. Cellular correlates of neuronal hyperexcitability in the vicinity of photochemically induced cortical infarcts in rats in vitro. Neurosci Lett. 1995;193(2):101-4.

27. Witte OW, Stoll G. Delayed and remote effects of focal cortical infarctions: secondary damage and reactive plasticity. Adv Neurol. 1997;73:207-27.

28. Bjorkman A, Rosen B, van Westen D, Larsson EM, Lundborg G. Acute improvement of contralateral hand function after deafferentation. Neuroreport. 2004:15(12):1861-5.

29. Mizelle JC, Oparah A, Wheaton LA. Reliability of visual and somatosensory feedback in skilled movement: the role of the cerebellum. Brain Topogr. 2016;29(1):27-41.

30. Beauchamp MS, Lee KE, Haxby JV, Martin A. Parallel visual motion processing streams for manipulable objects and human movements. Neuron. 2002;34(1):149-59.

31. Beauchamp MS, Laconte S, Yasar N. Distributed representation of single touches in somatosensory and visual cortex. Hum Brain Mapp. 2009:30(10):3163-71.

32. Oreja-Guevara C, Kleiser R, Paulus W, Kruse W, Seitz RJ, Hoffmann KP. The role of V5 (hMT+) in visually guided hand movements: an fMRI study. Eur J Neurosci. 2004;19(11):3113-20.

33. Kilner JM. More than one pathway to action understanding. Trends Cogn Sci. 2011;15(8):352-7.

34. Friston KJ, Daunizeau J, Kilner J, Kiebel SJ. Action and behavior: a free-energy formulation. Biol Cybern. 2010;102(3):227-60.
35. Shadmehr R, Smith MA, Krakauer JW. Error correction, sensory prediction, and adaptation in motor control. Annu Rev Neurosci. 2010;33:89-108.

36. Wolpert DM, Flanagan JR. Motor prediction. Curr Biol. 2001;11(18):R729-732.

37. Moisello C, Bove M, Huber R, Abbruzzese G, Battaglia F, Tononi G, Ghilardi MF. Short-term limb immobilization affects motor performance. J Mot Behav. 2008:40(2):165-76.

38. Bernier PM, Chua R, Bard C, Franks IM. Updating of an internal model without proprioception: a deafferentation study. Neuroreport. 2006;17(13):1421-5.

39. Philip BA, Frey SH. Stimulus-response correspondence across peripersonal space is unaffected by chronic unilateral limb loss. Exp Brain Res. 2013;224(3):373-82.

40. Philip BA, Frey SH. Preserved grip selection planning in chronic unilateral upper extremity amputees. Exp Brain Res. 2011;214(3):437-52.

41. Hashim E, Rowley CD, Grad S, Bock NA. Patterns of myeloarchitecture in lower limb amputees: an MRI study. Front Neurosci. 2015;9:15.

42. Jiang G, Yin X, Li C, Li L, Zhao L, Evans AC, Jiang T, Wu J, Wang J. The plasticity of brain gray matter and white matter following lower limb amputation. Neural Plast. 2015;2015:823185.

43. Xie H, Kane JT, Dennis MJ, Mooney RD, Bauer WR, Wang X, Wall JT. Case series evidence for changed interhemispheric relationships in cortical structure in some amputees. J Clin Neurosci. 2013;20(4):523-6.

44. Simoes EL, Bramati I, Rodrigues E, Franzoi A, Moll J, Lent R, Tovar-Moll F. Functional expansion of sensorimotor representation and structural reorganization of callosal connections in lower limb amputees. J Neurosci. 2012;32(9):3211-20.

45. Donoghue JP, Sanes JN. Organization of adult motor cortex representation patterns following neonatal forelimb nerve injury in rats. J Neurosci. 1988;8(9):3221-32

46. Wu CW, Kaas JH. Reorganization in primary motor cortex of primates with long-standing therapeutic amputations. J Neurosci. 1999;19(17):7679-97.

47. Pons TP, Garraghty PE, Ommaya AK, Kaas JH, Taub E, Mishkin M. Massive cortical reorganization after sensory deafferentation in adult macaques. Science. 1991;252(5014):1857-60.

48. Cohen LG, Bandinelli S, Findley TW, Hallett M. Motor reorganization after upper limb amputation in man. A study with focal magnetic stimulation. Brain. 1991;114(Pt 1B):615-27.

49. Knecht $\mathrm{S}$, Henningsen $\mathrm{H}$, Elbert T, Flor H, Hohling C, Pantev C, Taub E. Reorganizational and perceptional changes after amputation. Brain. 1996;119(Pt 4):1213-9.

50. Di Pino G, Guglielmelli E, Rossini PM. Neuroplasticity in amputees: main implications on bidirectional interfacing of cybernetic hand prostheses. Prog Neurobiol. 2009;88(2):114-26.

51. Mercier C, Reilly KT, Vargas CD, Aballea A, Sirigu A. Mapping phantom movement representations in the motor cortex of amputees. Brain. 2006;129(Pt 8):2202-10.

52. Reilly KT, Mercier C, Schieber MH, Sirigu A. Persistent hand motor commands in the amputees' brain. Brain. 2006;129(Pt 8):2211-23.

53. Flor H, Elbert T, Knecht S, Wienbruch C, Pantev C, Birbaumer N, Larbig W, Taub E. Phantom-limb pain as a perceptual correlate of cortical reorganization following arm amputation. Nature. 1995;375(6531):482-4.

54. Flor H, Nikolajsen L, Staehelin Jensen T. Phantom limb pain: a case of maladaptive CNS plasticity? Nat Rev Neurosci. 2006;7(11):873-81.

55. Flor H, Denke C, Schaefer M, Grusser S. Effect of sensory discrimination training on cortical reorganisation and phantom limb pain. Lancet. 2001;357(9270):1763-4.

56. Jenkins WM, Merzenich MM, Ochs MT, Allard T, Guic-Robles E. Functional reorganization of primary somatosensory cortex in adult owl monkeys after behaviorally controlled tactile stimulation. J Neurophysiol. 1990;63(1):82-104.

57. Pluto CP, Chiaia NL, Rhoades RW, Lane RD. Reducing contralateral SI activity reveals hindlimb receptive fields in the $\mathrm{SI}$ forelimb-stump representation of neonatally amputated rats. J Neurophysiol. 2005;94(3):1727-32.

58. Pluto CP, Lane RD, Rhoades RW. Local GABA receptor blockade reveals hindlimb responses in the SI forelimb-stump representation of neonatally amputated rats. J Neurophysiol. 2004;92(1):372-9.

59. Capaday C, Richardson MP, Rothwell JC, Brooks DJ. Long-term changes of GABAergic function in the sensorimotor cortex of amputees. A combined magnetic stimulation and 11C-flumazenil PET study. Exp Brain Res. 2000;133(4):552-6.

60. Makin TR, Filippini N, Duff EP, Henderson Slater D, Tracey I, Johansen-Berg H. Network-level reorganisation of functional connectivity following arm amputation. Neuroimage. 2015;114:217-25. 
61. Raichle ME. The brain's default mode network. Annu Rev Neurosci. 2015;38:433-47.

62. Van Overwalle F, Baetens K. Understanding others' actions and goals by mirror and mentalizing systems: a meta-analysis. Neuroimage. 2009;48(3):564-84.

63. Aziz-Zadeh L, Sheng T, Liew SL, Damasio H. Understanding otherness: the neural bases of action comprehension and pain empathy in a congenital amputee. Cereb Cortex. 2012;22(4):811-9.

64. Blank A, Okamura AM, Kuchenbecker KJ. Identifying the role of proprioception in upper-limb prosthesis control: Studies on targeted motion. ACM Trans Applied Perc. 2010;7(3):1-19.

65. Metzger AJ, Dromerick AW, Schabowsky CN, Holley RJ, Monroe B, Lum PS. Feedforward control strategies of subjects with transradial amputation in planar reaching. J Rehabil Res Dev. 2010;47(3):201-11.

66. Williams L, Pirouz N, Mizelle JC, Cusack W, Kistenberg R, Wheaton LA. Remodeling of cortical activity for motor control following upper limb loss. Clin Neurophysiol. 2016;127(9):3128-34.

67. Sammons RP, Keck T. Adult plasticity and cortical reorganization after peripheral lesions. Curr Opin Neurobiol. 2015;35:136-41.

68. Wu CW, Kaas JH. The effects of long-standing limb loss on anatomical reorganization of the somatosensory afferents in the brainstem and spinal cord. Somatosens Mot Res. 2002;19(2):153-63.

69. Wu CW, Kaas JH. Spinal cord atrophy and reorganization of motoneuron connections following long-standing limb loss in primates. Neuron. 2000;28(3):967-78.

70. Li CX, Yang Q, Vemulapalli S, Waters RS. Forelimb amputation-induced reorganization in the cuneate nucleus (CN) is not reflected in large-scale reorganization in rat forepaw barrel subfield cortex (FBS). Brain Res. 2013;1526:26-43.

71. Lane RD, Pluto CP, Kenmuir CL, Chiaia NL, Mooney RD. Does reorganization in the cuneate nucleus following neonatal forelimb amputation influence development of anomalous circuits within the somatosensory cortex? J Neurophysiol. 2008;99(2):866-75.

72. Li CX, Chappell TD, Ramshur JT, Waters RS. Forelimb amputation-induced reorganization in the ventral posterior lateral nucleus (VPL) provides a substrate for large-scale cortical reorganization in rat forepaw barrel subfield (FBS). Brain Res. 2014;1583:89-108.

73. Stojic AS, Lane RD, Killackey HP, Qadri BA, Rhoades RW. Thalamocortical and intracortical projections to the forelimb-stump SI representation of rats that sustained neonatal forelimb removal. J Comp Neurol. 1998;401(2):187-204.

74. Biane JS, Takashima Y, Scanziani M, Conner JM, Tuszynski MH. Thalamocortical projections onto behaviorally relevant neurons exhibit plasticity during adult motor learning. Neuron. 2016;89(6):1173-9.

75. Stepniewska I, Preuss TM, Kaas JH. Thalamic connections of the dorsal and ventral premotor areas in New World owl monkeys. Neuroscience. 2007;147(3):727-45.

76. Gharbawie OA, Stepniewska I, Burish MJ, Kaas JH. Thalamocortical connections of functional zones in posterior parietal cortex and frontal cortex motor regions in New World monkeys. Cereb Cortex. 2010;20(10):2391-410

77. Percheron G, Francois C, Talbi B, Yelnik J, Fenelon G. The primate motor thalamus. Brain Res Brain Res Rev. 1996;22(2):93-181.

78. Banati RB, Cagnin A, Brooks DJ, Gunn RN, Myers R, Jones T, Birch R, Anand P. Long-term trans-synaptic glial responses in the human thalamus after peripheral nerve injury. Neuroreport. 2001;12(16):3439-42.

79. Pluto CP, Lane RD, Chiaia NL, Stojic AS, Rhoades RW. Role of development in reorganization of the $\mathrm{SI}$ forelimb-stump representation in fetally, neonatally, and adult amputated rats. J Neurophysiol. 2003;90(3):1842-51.

80. Paradiso G, Cunic D, Saint-Cyr JA, Hoque T, Lozano AM, Lang AE, Chen R. Involvement of human thalamus in the preparation of self-paced movement. Brain. 2004;127(Pt 12):2717-31.

81. Schofield JS, Evans KR, Carey JP, Herbert PS. Applicaitons of sensory feedback in motorized upper extremity prostheses: a review. Expert Rev Med Devices. 2014;11(5):499-501.

82. Clancy KB, Koralek AC, Costa RM, Feldman DE, Carmena JM. Volitional modulation of optically recorded calcium signals during neuroprosthetic learning. Nat Neurosci. 2014;17(6):807-9.

83. de Jong BM, Coert JH, Stenekes MW, Leenders KL, Paans AM, Nicolai JP. Cerebral reorganisation of human hand movement following dynamic immobilisation. Neuroreport. 2003;14(13):1693-6.

84. Stenekes MW, Coert JH, Nicolai JP, Mulder T, Geertzen JH, Paans AM, de Jong BM. Cerebral consequences of dynamic immobilisation after primary digital flexor tendon repair. J Plast Reconstr Aesthet Surg. 2010;63(12):1953-61.

85. Avanzino L, Pelosin E, Abbruzzese G, Bassolino M, Pozzo T, Bove M. Shaping motor cortex plasticity through proprioception. Cereb Cortex. 2014;24(10):2807-14

86. Schwenkreis $P$, Witscher $K$, Janssen F, Pleger B, Dertwinkel R, Zenz M, Malin JP, Tegenthoff M. Assessment of reorganization in the sensorimotor cortex after upper limb amputation. Clin Neurophysiol. 2001;112(4):627-35.

87. Schwenkreis P, Witscher K, Janssen F, Dertwinkel R, Zenz M, Malin JP, Tegenthoff M. Changes of cortical excitability in patients with upper limb amputation. Neurosci Lett. 2000;293(2):143-6.

88. Garry MI, Kamen G, Nordstrom MA. Hemispheric differences in the relationship between corticomotor excitability changes following a fine-motor task and motor learning. J Neurophysiol. 2004;91(4):1570-8.

89. Kobayashi M, Theoret H, Pascual-Leone A. Suppression of ipsilateral motor cortex facilitates motor skill learning. Eur J Neurosci. 2009;29(4):833-6.

90. Hughey L, Wheaton LA. Implicit learning and explicit recall in users of upper extremity prostheses: insights into functional rehabilitation challenges. J Mot Behav. In press.

91. Marini F, Tagliabue CF, Sposito AV, Hernandez-Arieta A, Brugger P, Estevez N, Maravita A. Crossmodal representation of a functional robotic hand arises after extensive training in healthy participants. Neuropsychologia. 2014:53:178-86.

92. Chai G, Sui X, Li S, He L, Lan N. Characterization of evoked tactile sensation in forearm amputees with transcutaneous electrical nerve stimulation. J Neural Eng. 2015;12(6):066002.

93. Chen A, Yao J, Kuiken T, Dewald JP. Cortical motor activity and reorganization following upper-limb amputation and subsequent targeted reinnervation. Neurolmage Clin. 2013;3:498-506.

94. Hebert JS, Chan KM, Dawson MR. Cutaneous sensory outcomes from three transhumeral targeted reinnervation cases. Prosthet Orthot Int. 2016:40(3):303-10.

95. Kuiken TA, Miller LA, Lipschutz RD, Lock BA, Stubblefield K, Marasco PD, Zhou P, Dumanian GA. Targeted reinnervation for enhanced prosthetic arm function in a woman with a proximal amputation: a case study. Lancet. 2007:369(9559):371-80.

96. Miller LA, Stubblefield KA, Lipschutz RD, Lock BA, Kuiken TA. Improved myoelectric prosthesis control using targeted reinnervation surgery: a case series. IEEE Trans Neural Syst Rehabil Eng. 2008;16(1):46-50.

97. Bergmeister KD, Hader M, Lewis S, Russold MF, Schiestl M, Manzano-Szalai K, Roche AD, Salminger S, Dietl H, Aszmann OC. Prosthesis control with an implantable multichannel wireless electromyography system for high-level amputees: a large-animal study. Plast Reconstr Surg. 2016;137(1):153-62.

98. Pasquina PF, Evangelista M, Carvalho AJ, Lockhart J, Griffin S, Nanos G, McKay P, Hansen M, Ipsen D, Vandersea J, et al. First-in-man demonstration of a fully implanted myoelectric sensors system to control an advanced electromechanical prosthetic hand. J Neurosci Methods. 2015;244:85-93.

99. Cipriani C, Antfolk C, Controzzi M, Lundborg G, Rosen B, Carrozza MC, Sebelius F. Online myoelectric control of a dexterous hand prosthesis by transradial amputees. IEEE Trans Neural Syst Rehabil Eng. 2011;19(3):260-70.

100. Smith LH, Kuiken TA, Hargrove LJ. Real-time simultaneous and proportional myoelectric control using intramuscular EMG. J Neural Eng. 2014;11(6):066013.

101. Kapelner T, Jiang N, Holobar A, Vujaklija I, Roche AD, Farina D, Aszmann OC. Motor unit characteristics after targeted muscle reinnervation. PLoS One. 2016;11(2):e0149772.

102. Miller LA, Lipschutz RD, Stubblefield KA, Lock BA, Huang H, Williams 3rd TW, Weir RF, Kuiken TA. Control of a six degree of freedom prosthetic arm after targeted muscle reinnervation surgery. Arch Phys Med Rehabil. 2008:89(11):2057-65.

103. Graczyk EL, Schiefer MA, Saal HP, Delhaye BP, Bensmaia SJ, Tyler DJ. The neural basis of perceived intensity in natural and artificial touch. Sci Transl Med. 2016:8(362):362ra142

104. Hasson CJ, Manczurowsky J. Effects of kinematic vibrotactile feedback on learning to control a virtual prosthetic arm. J Neuroeng Rehabil. 2015;12:31.

105. Brown JD, Paek A, Syed M, O'Malley MK, Shewokis PA, Contreras-Vidal JL, Davis AJ, Gillespie RB. An exploration of grip force regulation with a low-impedance myoelectric prosthesis featuring referred haptic feedback. J Neuroeng Rehabil. 2015;12:104.

106. Bouwsema H, van der Sluis CK, Bongers RM. Effect of feedback during virtual training of grip force control with a myoelectric prosthesis. PLoS One. 2014;9(5):e98301. 
107. Stepp CE, Matsuoka Y. Vibrotactile sensory substitution for object manipulation: amplitude versus pulse train frequency modulation. IEEE Trans Neural Syst Rehabil Eng. 2012;20(1):31-7.

108. Childress DS. Closed-loop control in prosthetic systems: historical perspective. Ann Biomed Eng. 1980;8(4-6):293-303.

109. Brown J, Kunz T, Gardner D, Shelley M, Davis A, Gillespie B. An Empirical Evaluation of Force Feedback in Body-Powered Prostheses. IEEE Trans Neural Syst Rehabil Eng. 2016;25(3):215-26.

110. Umilta MA, Escola L, Intskirveli I, Grammont F, Rochat M, Caruana F, Jezzini A, Gallese V, Rizzolatti $G$. When pliers become fingers in the monkey motor system. Proc Natl Acad Sci U S A. 2008;105(6):2209-13.

111. Botvinick M, Cohen J. Rubber hands 'feel' touch that eyes see. Nature. 1998;391(6669):756.

112. Armel KC, Ramachandran VS. Projecting sensations to external objects: evidence from skin conductance response. Proc Biol Sci. 2003;270(1523):1499-506.

113. Ehrsson HH, Rosen B, Stockselius A, Ragno C, Kohler P, Lundborg G. Upper limb amputees can be induced to experience a rubber hand as their own. Brain. 2008;131(Pt 12):3443-52.

114. Ehrsson HH, Spence C, Passingham RE. That's my hand! Activity in premotor cortex reflects feeling of ownership of a limb. Science. 2004;305(5685):875-7.

115. Ehrsson HH, Holmes NP, Passingham RE. Touching a rubber hand: feeling of body ownership is associated with activity in multisensory brain areas. J Neurosci. 2005;25(45):10564-73.

116. Tsakiris M. My body in the brain: a neurocognitive model of body-ownership. Neuropsychologia. 2010;48(3):703-12

117. Guterstam A, Petkova Vl, Ehrsson $\mathrm{HH}$. The illusion of owning a third arm. PLoS One. 2011;6(2):e17208.

118. Cusack WF, Cope M, Nathanson S, Pirouz N, Kistenberg RS, Wheaton LA. Neural activation differences in amputees during imitation of intact versus amputee movements. Front Human Neurosci. 2012;6:182.

119. Deeny SP, Haufler AJ, Saffer M, Hatfield BD. Electroencephalographic coherence during visuomotor performance: a comparison of cortico-cortical communication in experts and novices. J Mot Behav. 2009;41(2):106-16.

120. Quallo MM, Price CJ, Ueno K, Asamizuya T, Cheng K, Lemon RN, Iriki A. Gray and white matter changes associated with tool-use learning in macaque monkeys. Proc Natl Acad Sci U S A. 2009;106(43):18379-84.

121. Vingerhoets $G$. Knowing about tools: neural correlates of tool familiarity and experience. Neuroimage. 2008;40(3):1380-91.

122. Mizelle JC, Tang T, Pirouz N, Wheaton LA. Forming Tool Use Representations: A Neurophysiological Investigation into Tool Exposure. J Cogn Neurosci. 2011;23(10):2920-34.

123. Nico D, Daprati E, Rigal F, Parsons L, Sirigu A. Left and right hand recognition in upper limb amputees. Brain. 2004;127(Pt 1):120-32.

124. Shah B, Nguyen TT, Madhavan S. Polarity independent effects of cerebellar tDCS on short term ankle visuomotor learning. Brain Stimul. 2013;6(6):966-8.

125. Dutta A, Paulus W, Nitsche MA. Facilitating myoelectric-control with transcranial direct current stimulation: a preliminary study in healthy humans. J Neuroeng Rehabil. 2014;11(1):13.

126. Cusack WF, Patterson R, Thach S, Kistenberg RS, Wheaton LA. Motor performance benefits of matched limb imitation in prosthesis users. Exp Brain Res. 2014;232:2143-54.

127. Lawson DT, Cusack WF, Lawson R, Hardy A, Kistenberg R, Wheaton LA Influence of perspective of action observation training on residual limb control in naive prosthesis usage. J Mot Behav. 2016;48(5):446-54.

128. Weeks DL, Wallace SA, Anderson DI. Training with an upper-limb prosthetic simulator to enhance transfer of skill across limbs. Arch Phys Med Rehabil. 2003:84(3):437-43.

129. de Boer E, Romkema S, Cutti AG, Brouwers MA, Bongers RM, van der Sluis CK. Intermanual Transfer Effects in Below-Elbow Myoelectric Prosthesis Users. Arch Phys Med Rehabil. 2016;97(11):1924-30.

130. Romkema S, Bongers RM, van der Sluis CK. Intermanual transfer effect in young children after training in a complex skill: mechanistic, pseudorandomized, pretest-posttest study. Phys Ther. 2015;95(5):730-9.

131. Makin TR, Cramer AO, Scholz J, Hahamy A, Henderson Slater D, Tracey I, Johansen-Berg H. Deprivation-related and use-dependent plasticity go hand in hand. Elife. 2013;2:e01273.

132. Resnik L, Etter K, Klinger SL, Kambe C. Using virtual reality environment to facilitate training with advanced upper-limb prosthesis. J Rehabil Res Dev. 2011:48(6):707-18
133. Spiegel DR, Lappinen E, Gottlieb M. A presumed case of phantom limb pain treated successfully with duloxetine and pregabalin. Gen Hosp Psychiatry. 2010;32(2):228. e225-227.

134. Greer TL, Sunderajan P, Grannemann BD, Kurian BT, Trivedi MH. Does duloxetine improve cognitive function independently of its antidepressant effect in patients with major depressive disorder and subjective reports of cognitive dysfunction? Depress Res Treat. 2014;2014:627863.

135. Stagg CJ, Bachtiar $V$, Johansen-Berg $H$. The role of GABA in human motor learning. Curr Biol. 2011;21(6):480-4

136. Smurr LM, Gulick K, Yancosek K, Ganz O. Managing the upper extremity amputee: a protocol for success. J Hand Ther. 2008;21(2):160-75. quiz 176.

137. Ephraim PL, Dillingham TR, Sector M, Pezzin LE, Mackenzie EJ. Epidemiology of limb loss and congenital limb deficiency: a review of the literature. Arch Phys Med Rehabil. 2003;84(5):747-61.

138. Salminger S, Gradischar A, Skiera R, Roche AD, Sturma A, Hofer C, Aszmann OC. Attachment of upper arm prostheses with a subcutaneous osseointegrated implant in transhumeral amputees. Prosthet Orthot Int. 2016.

139. Solarz MK, Thoder JJ, Rehman S. Management of major traumatic upper extremity amputations. Orthop Clin North Am. 2016;47(1):127-36.

140. Reinkensmeyer DJ, Burdet E, Casadio M, Krakauer JW, Kwakkel G, Lang CE, Swinnen SP, Ward NS, Schweighofer N. Computational neurorehabilitation: modeling plasticity and learning to predict recovery. J Neuroeng Rehabil. 2016;13(1):42.

\section{Submit your next manuscript to BioMed Central and we will help you at every step:}

- We accept pre-submission inquiries

- Our selector tool helps you to find the most relevant journal

- We provide round the clock customer support

- Convenient online submission

- Thorough peer review

- Inclusion in PubMed and all major indexing services

- Maximum visibility for your research

Submit your manuscript at www.biomedcentral.com/submit

) Biomed Central 